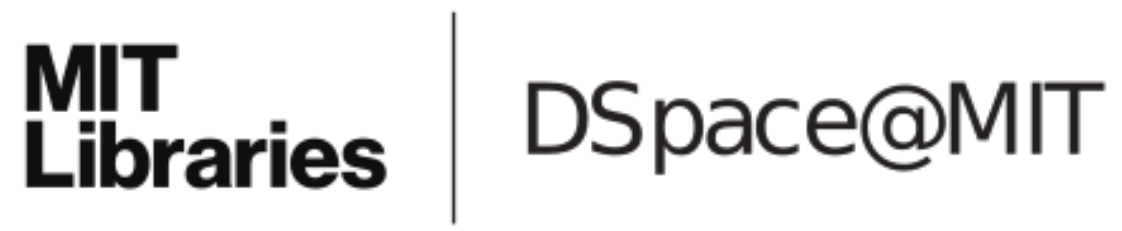

\author{
MIT Open Access Articles
}

\begin{abstract}
ON THE GEOMETRIC NATURE OF LOWFREQUENCY QUASI-PERIODIC OSCILLATIONS IN NEUTRON-STAR LOW-MASS X-RAY BINARIES
\end{abstract}

The MIT Faculty has made this article openly available. Please share how this access benefits you. Your story matters.

Citation: Homan, Jeroen, Joel K. Fridriksson, and Ronald A. Remillard. "ON THE GEOMETRIC NATURE OF LOW-FREQUENCY QUASI-PERIODIC OSCILLATIONS IN NEUTRON-STAR LOWMASS X-RAY BINARIES." The Astrophysical Journal 812, no. 1 (October 9, 2015): 80. (c) 2015 The American Astronomical Society

As Published: http://dx.doi.org/10.1088/0004-637X/812/1/80

Publisher: IOP Publishing

Persistent URL: http://hdl.handle.net/1721.1/99905

Version: Final published version: final published article, as it appeared in a journal, conference proceedings, or other formally published context

Terms of Use: Article is made available in accordance with the publisher's policy and may be subject to US copyright law. Please refer to the publisher's site for terms of use. 


\title{
ON THE GEOMETRIC NATURE OF LOW-FREQUENCY QUASI-PERIODIC OSCILLATIONS IN NEUTRON-STAR LOW-MASS X-RAY BINARIES
}

\author{
Jeroen Homan $^{1,2}$, Joel K. Fridriksson ${ }^{3}$, and Ronald A. Remillard ${ }^{1}$ \\ ${ }^{1}$ MIT Kavli Institute for Astrophysics and Space Research, 77 Massachusetts Avenue 37-582D, Cambridge, MA 02139, USA; jeroen@space.mit.edu \\ ${ }^{2}$ SRON, Netherlands Institute for Space Research, Sorbonnelaan 2, 3584 CA Utrecht, The Netherlands \\ 3 Anton Pannekoek Institute for Astronomy, University of Amsterdam, Postbus 94249, 1090 GE Amsterdam, The Netherlands \\ Received 2015 July 21; accepted 2015 August 28; published 2015 October 9
}

\begin{abstract}
We report on a detailed analysis of the so-called $\sim 1 \mathrm{~Hz}$ quasi-periodic oscillation (QPO) in the eclipsing and dipping neutron-star low-mass X-ray binary EXO 0748-676. This type of QPO has previously been shown to have a geometric origin. Our study focuses on the evolution of the QPO as the source moves through the color-color diagram in which it traces out an atoll-source-like track. The QPO frequency increases from $\sim 0.4 \mathrm{~Hz}$ in the hard state to $\sim 25 \mathrm{~Hz}$ as the source approaches the soft state. Combining power spectra based on QPO frequency reveals additional features that strongly resemble those seen in non-dipping/eclipsing atoll sources. We show that the lowfrequency QPOs in atoll sources and the $\sim 1 \mathrm{~Hz}$ QPO in EXO 0748-676 follow similar relations with respect to the noise components in their power spectra. We conclude that the frequencies of both types of QPOs are likely set by (the same) precession of a misaligned inner accretion disk. For high-inclination systems like EXO 0748-676 this results in modulations of the neutron-star emission due to obscuration or scattering, while for lower-inclination systems the modulations likely arise from relativistic Doppler-boosting and light-bending effects.
\end{abstract}

Key words: accretion, accretion disks - stars: neutron - X-rays: binaries - X-rays: individual (EXO 0748-676)

\section{INTRODUCTION}

Low-frequency quasi-periodic oscillations (LF-QPOs) are a common phenomenon in the X-ray light curves of low-mass $\mathrm{X}$-ray binaries. In power spectra they show up as narrow peaks with frequencies ranging from $\sim 1$ to $\sim 70 \mathrm{~Hz}$ in neutron-star low-mass X-ray binaries (NS-LMXBs) and from $\sim 0.01$ to $\sim 30 \mathrm{~Hz}$ in black hole low-mass X-ray binaries (BH-LMXBs). Comparisons of the power spectra of NS-LMXBs and BHLMXBs suggest that the most common types of LF-QPOs in these systems (as well as some of the other variability components) may have a similar origin (Psaltis et al. 1999; Wijnands \& van der Klis 1999; Belloni et al. 2002).

For the most common type of LF-QPOs in BH-LMXBs, the so-called type-C QPOs (Casella et al. 2005), there is increasing evidence that points toward a geometric origin: these QPOs are stronger in sources that are viewed at higher inclinations (Schnittman et al. 2006; Heil et al. 2015; Motta et al. 2015), and the strength of the $\mathrm{Fe} \alpha$ emission line in GRS 1915+105 has been found to be strongly modulated as part of the type-C QPO cycle (Miller \& Homan 2005; Ingram \& van der Klis 2015). Both these results are consistent with the QPO being generated by a misaligned and precessing inner accretion disk (Schnittman et al. 2006), with relativistic Lense-Thirring precession being a commonly suggested mechanism (e.g., Ingram et al. 2009).

In seven dipping and/or eclipsing NS-LMXBs a type of LFQPO has been observed whose properties suggest (even more directly) that it also has a geometric origin (Homan 2012). These QPOs are often referred to as the " $\sim 1 \mathrm{~Hz}$ QPOs," due to the fact that they were initially discovered around $1 \mathrm{~Hz}$ in three different sources (Homan et al. 1999; Jonker et al. 1999, 2000). The geometric nature of these QPOs was deduced from the fact that these QPOs persist during type I (thermonuclear) X-ray bursts with a fractional amplitude that is consistent with that observed during the persistent (non-burst) emission, and that their strength is nearly independent of energy. Both these properties suggests that (part of) the emission from the neutron star is obscured or scattered out of the line of sight by the accretion flow. Homan (2012) recently suggested that an inclined and precessing inner accretion flow might be a possible explanation for the observed properties of the $\sim 1 \mathrm{~Hz}$ QPOs. The misalignment angle between the inner flow and binary plane would have to be $\sim 15^{\circ}-20^{\circ}$ for this type of QPO to be observed only in dipping and/or eclipsing NS-LMXBs. The relation between these $\sim 1 \mathrm{~Hz}$ QPOs and the LF-QPOs observed in the non-dipping/eclipsing NS-LMXB is not clear at this point. Although Homan (2012) pointed out some similarities (e.g., both being mainly observed in the harder spectral states) the $\sim 1 \mathrm{~Hz}$ QPOs in the dipping/eclipsing sources have not been studied in enough detail to make a proper comparison.

In this paper we investigate the $\sim 1 \mathrm{~Hz}$ QPO observed in the dipping and eclipsing NS-LMXB EXO 0748-676. Of the seven sources in which $\sim 1 \mathrm{~Hz}$ QPOs have been observed, EXO 0748-676 has the largest reported frequency range. It also has the highest number of Rossi X-ray Timing Explorer (RXTE) observations and is known to make regular state transitions. We focus on how the $\sim 1 \mathrm{~Hz}$ QPO evolves along the tracks traced out in the color-color diagram (CD; Section 3.1) and perform a comparison of the observed power spectra with those of non-dipping/eclipsing NS-LMXBs (Section 3.2). Our results are discussed in Section 4.

\section{OBSERVATIONS AND DATA REDUCTION}

The observations of EXO 0748-676 analyzed in this paper were made with the Proportional Counter Array (PCA; Jahoda et al. 2006) on board RXTE (Bradt et al. 1993). EXO 0748-676 was observed 752 times with the PCA during the lifetime of RXTE. A number of observations had to be discarded for various reasons: they were made after the source had returned to quiescence (Wolff et al. 2008a, 2008b), important data modes were missing, or not enough data were left to produce 
power spectra of the required length (128 s) after the recommended filtering criteria had been applied. This left 718 useful observations. To increase the sensitivity to QPOs, observations were combined into groups. Consecutive observations were added together if the time gap between them was less than $12 \mathrm{hr}$, resulting in 203 groups of observations. In all almost all cases these groups covered periods less than a day, but for seven groups the period was longer (up to $\sim 2$ days). These seven groups were split into two or three shorter segments, covering less than a day. The final number of groups was 211, with individual groups containing between 1 and 11 observations. The group exposure times ranged from $\sim 350 \mathrm{~s}$ to $\sim 40 \mathrm{ks}$, with an average of $\sim 8 \mathrm{ks}$. Note that this process of combining observations is justified by the fact that the $\sim 1 \mathrm{~Hz}$ QPO in EXO 0748-676 was seen to vary in frequency by less than $\sim 15 \%$ on a timescale of a day (Homan et al. 1999).

We used standard 2 mode data to create light curves, hardness-intensity diagrams (HIDs), and CDs. Data from all available Proportional Counter Units (PCUs) were used. Following the procedures laid out in Fridriksson et al. (2015), count rates from each PCU were corrected for changes in the response, using long-term light curves of the Crab, and then normalized to those of PCU 2. We define a soft color as the net counts in the 4.0-7.3 keV band divided by those in the $2.4-4.0 \mathrm{keV}$ band, and a hard color (hardness) as the net counts in the $9.8-18.2 \mathrm{keV}$ band divided by those in the $7.3-9.8 \mathrm{keV}$ band. The intensity we use for the HIDs is the net count rate per PCU in the $2-60 \mathrm{keV}$ band. The standard 2 data were barycenter corrected and eclipses were removed using the ephemeris from Wolff et al. (2009). Type I X-ray bursts were removed using standard 1 mode data, following the procedures laid out in Remillard et al. (2006). The standard 2 and high-time-resolution data were filtered following a similar procedure. Absorption dips were removed from the standard 2 data, since they strongly affect the average count rates and colors of the observations. The removal of dips had to be done manually and was based on visual inspection of the light curves and soft-color curves. Given the strong differences between observations in terms of dipping activity it was difficult to remove dips in a fully consistent manner for the entire data set. We did not remove the dips from the high-timeresolution data used for the creation of the power spectra, since the $\sim 1 \mathrm{~Hz}$ QPO can still be detected during dips (Homan et al. 1999).

Power spectra were created from light curves extracted from the high-time-resolution data modes. Light curves were created with a time resolution of $1 / 8192 \mathrm{~s}$ (except for the first observation, 10068-03-01-00, which had a maximum time resolution of $1 / 2048 \mathrm{~s}$ ), covering absolute PCA channels 0-35. This channel band effectively corresponds to an energy range of $\sim 1.5-9.5 \mathrm{keV}$ during Gain Epoch 1, increasing to $\sim 2.1-15 \mathrm{keV}$ during Gain Epochs 4 and 5. We note that more than 600 of our observations were made during Gain Epochs 4 and 5. To create the power spectra we performed Fast Fourier Transformations of $128 \mathrm{~s}$ light-curve segments. The power spectra were averaged for each group and rms normalized (Belloni \& Hasinger 1990; Miyamoto et al. 1991; van der Klis 1995). These normalized power spectra were rebinned logarithmically to 70 bins per frequency decade; for plotting purposes the power spectra were sometimes rebinned even further. Prior to fitting we subtracted the deadtime-modified Poisson level, which we approximated by taking the average

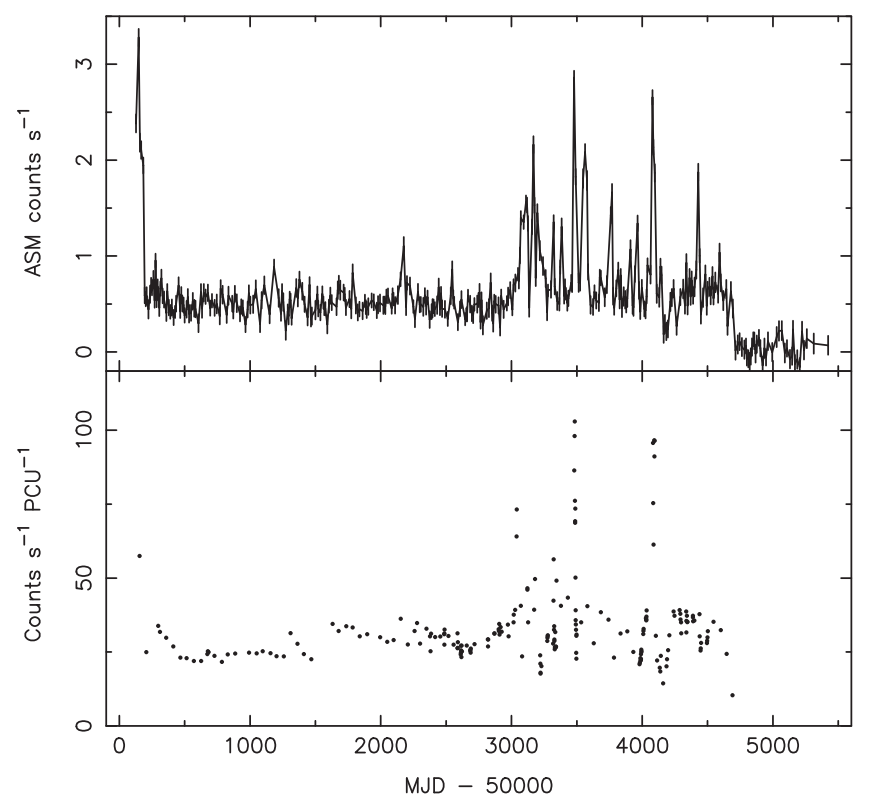

Figure 1. Long-term light curves of EXO 0748-676. In the top panel we show seven-day averages from the RXTE/ASM $(\sim 1.5-12 \mathrm{keV})$. In the bottom panel we show data from the RXTE/PCA $(\sim 2-60 \mathrm{keV})$, with each data point representing the average count rate of a single group. Errors on the PCA count rates are smaller than the size of the plot symbols. Around MJD 54700 (2008 August) the source returned to quiescence.

power in an interval of a few $100 \mathrm{~Hz}$ centered around $\sim 2000 \mathrm{~Hz}$. While this method may introduce some uncertainties in the properties of the high-frequency features, it should have a negligible effect on the low-frequency features in the power spectra (which are the focus of this paper). The resulting power spectra were fitted up to $2000 \mathrm{~Hz}$ with a combination of a power law $\left(P(\nu) \propto \nu^{-\alpha}\right)$, and one or more Lorentzians $\left(P(\nu)=\left(r^{2} \Delta / \pi\right)\left[\Delta^{2}+\left(\nu-\nu_{0}\right)^{2}\right]^{-1}\right)$. Here $\nu_{0}$ is the centroid frequency, $\Delta$ the half-width at half-maximum, and $r$ the integrated fractional rms (from $-\infty$ to $\infty$ ). Instead of $\nu_{0}$ and $\Delta$ we will quote the frequency $\nu_{\max }$ at which the Lorentzian attains its maximum in $\nu P(\nu)$ and the quality factor, $\mathrm{Q}$, where $\nu_{\max }=\nu_{0}\left(1+1 / 4 Q^{2}\right)^{1 / 2}$ and $Q=\nu_{0} / 2 \Delta$ (Belloni et al. 2002). The fractional rms amplitudes quoted in this paper were the integrated power between 0 and $\infty \mathrm{Hz}$ for the Lorentzians, and between 0.01 and $100 \mathrm{~Hz}$ for the power-law component. Errors on fit parameters were determined using $\Delta \chi^{2}=1$ (i.e., $68 \%$ confidence).

\section{ANALYSIS \& RESULTS}

\subsection{The $\sim 1 \mathrm{~Hz} Q P O$ in EXO 0748-676}

In Figure 1 we show long-term light curves of EXO 0748-676 from the RXTE All-Sky Monitor ${ }^{4}$ (ASM; Levine et al. 1996) and the PCA. The ASM light curve shows 7-day averages, while the PCA light curve shows the average count rate for each group. Especially during the second half of RXTE's lifetime EXO 0748-676 was quite variable, with occasional several-week-long outbursts. In Figure 2 we show the HID and CD corresponding to the PCA light curve. The patterns traced out in those diagrams are very similar to those of some of the low-luminosity (atoll) NS-LMXBs (see, e.g., Gladstone et al. (2007) and Fridriksson (2011) for collections

\footnotetext{
4 Public ASM data were obtained from http://xte.mit.edu.
} 


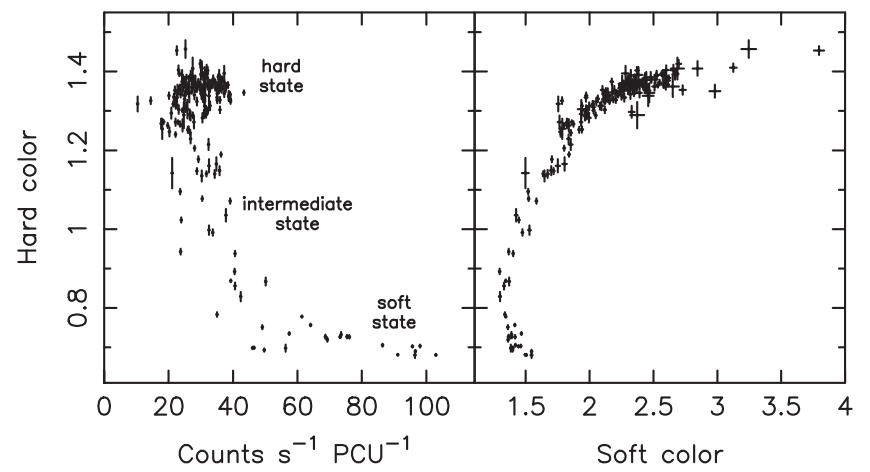

Figure 2. Hardness-intensity diagram (left) and color-color diagram (right) of EXO 0748-676. Each data point represents the average of a single group. See Section 2 for definition of colors.

of atoll-source CDs/HIDs). As already pointed out by Ponti et al. (2014) and Muñoz-Darias et al. (2014), hard and soft states can be identified in the CD/HID of EXO 0748-676. In the HID in Figure 2 we label the hard, intermediate, and soft states; these states are often also referred to as the extreme island state, the island state and the (lower) banana state, respectively. A comparison between the CD of EXO 0748-676 and those of the atoll sources presented in Fridriksson (2011) indicates that the soft color of EXO 0748-676 spans an unusually large range. This is likely the result of incomplete dip removal, which mostly affects that low energy bands. We note that before the dip removal the soft color range was $\sim 1.3-5.1$.

Based on a visual inspection of all 211 power spectra for the presence of a $\sim 1 \mathrm{~Hz}$ QPO, we selected 171 for further analysis. Following the analysis of Homan et al. (1999) these 171 power spectra were initially fit with a combination of a power law and a single Lorentzian. While in most cases this model resulted in acceptable fits (average $\chi_{\text {red }}^{2} \approx 1.18$ ), in some power spectra the QPO profile was not accurately fit because of a broad underlying noise component. Adding a zero-centered Lorentzian to the fit function solved this issue and resulted in an average $\chi_{\text {red }}^{2}$ of $\sim 1.05$. QPOs with single-trial significances 5 larger than $3 \sigma$ were detected in the power spectra of 157 groups, with frequencies ranging from $\sim 0.43$ to $\sim 25.5 \mathrm{~Hz}$. Together these 157 groups represent $558(\sim 78 \%)$ of the 718 observations we analyzed.

To see in which states the $\sim 1 \mathrm{~Hz}$ QPO was detected we show a color-coded CD in Figure 3; the groups with LF-QPO detections are colored red, and those without are colored gray. It is clear from this figure that the $\sim 1 \mathrm{~Hz}$ QPO was predominantly detected in the hard state, with a few detections in the intermediate state. In order to study how the QPO properties evolve along the track in the $\mathrm{CD}$, we parameterized the position along the track by manually drawing a spline onto which all data points were projected (see, e.g., van der Klis 2000). To correct for the differences in scale between the horizontal and vertical axes in Figure 3, the soft-color values were scaled down by a factor of 0.286 during the projection procedure, thus ensuring that the data points were projected onto the nearest spline point in the figure. Two normal points, depicted by the large black circles in Figure 3, were chosen to set the scale for the track parameter $S_{\mathrm{a}} . S_{\mathrm{a}}=1$ was chosen to fall close to the end of the dense cluster of points in the hard state of EXO 0748-676, while $S_{\mathrm{a}}=2$ was chosen to coincide

\footnotetext{
The integrated power divided by its negative error.
}

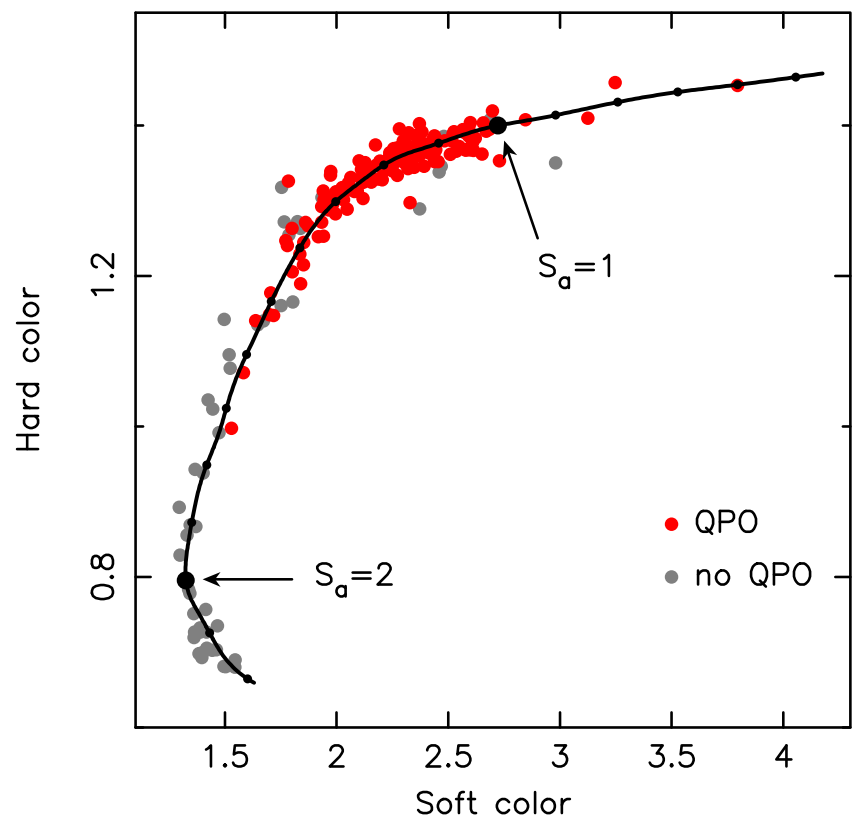

Figure 3. Color-color diagram of EXO 0748-676, showing groups with $\sim 1 \mathrm{~Hz}$ QPO detections (red) and those without (gray). The black line shows the spline used to calculate the $S_{\mathrm{a}}$ values for each data point. The two large black circles indicate the locations of $S_{\mathrm{a}}=1$ and $S_{\mathrm{a}}=2$. The smaller black circles represent steps in $S_{\mathrm{a}}$ of 0.1 . Error bars are omitted for clarity.

with the boundary of the intermediate state and the soft state (most clearly seen in the HID in Figure 2; hard color $\sim 0.8$ ). The smaller black circles represent steps of 0.1 in $S_{\mathrm{a}}$. Using this parametrization we find that no $\sim 1 \mathrm{~Hz}$ QPO was detected significantly for $S_{\mathrm{a}}$ values greater than 1.73 . Note that the observation that showed the $695 \mathrm{~Hz}$ QPO reported by Homan $\&$ van der Klis (2000) has an $S_{\mathrm{a}}$ value of $\sim 2.1$.

In Figure 4 we show the QPO frequency, $Q$-value and rms amplitude as a function of $S_{\mathrm{a}}$. The QPO frequency shows a clear increase with $S_{\mathrm{a}}$, although there is considerable scatter at low $S_{\mathrm{a}}$ values. The $Q$-value shows no clear dependence on $S_{\mathrm{a}}$, while the rms appears to increase toward higher $S_{\mathrm{a}}$.

Part of the scatter observed in Figure 4 is likely caused by the fact that we were not able to perfectly remove all dipping intervals from the data. Since dipping is most prevalent in the hard state (i.e., at low $S_{\mathrm{a}}$ ) and increases the soft color, the removal of dips will move observations parallel to the spline in the $\mathrm{CD}$, toward higher $S_{\mathrm{a}}$. Various gradations of incomplete dip removal will therefore result in a range of $S_{\text {a }}$ values for observations whose non-dipping emission would have the same $S_{\mathrm{a}}$ value. To reduce the impact from these effects, and also to boost the signal-to-noise ratio of our power spectra, we performed additional grouping of our observations. Observations from groups with similar QPO frequencies were combined into 21 new groups. At the low-frequency end the width of the frequency selections for the new groups was $0.1 \mathrm{~Hz}$, increasing to larger values for higher frequencies; the gray horizontal lines in the top panel of Figure 4 indicate the boundaries separating the 21 frequency-based groups. These new groups contained between 1 and 17 of the old groups, and between 1 and 69 of the original observations. To determine $S_{\text {a }}$ values for each of the new groups we reprojected the averaged CD data points onto the previously used spline.

As a result of the increased signal-to-noise we were able to detect additional features in the power spectra, especially for 


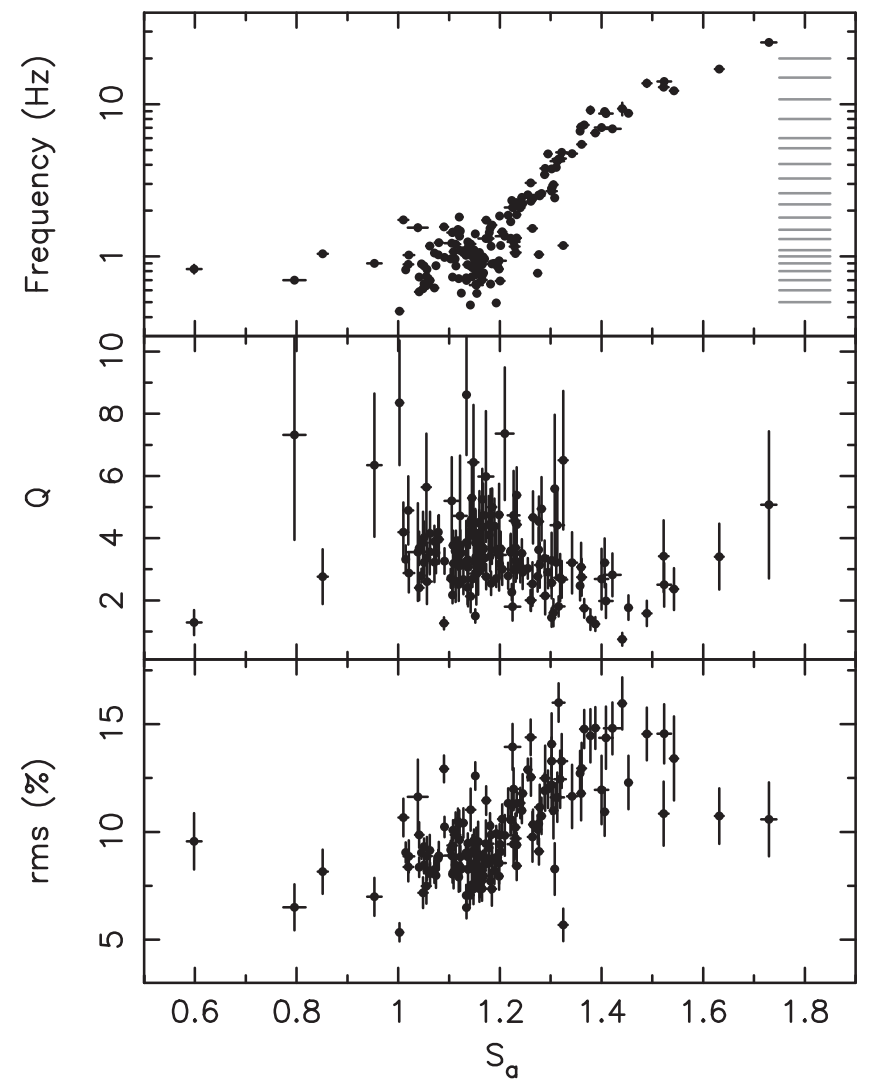

Figure 4. Properties of the $\sim 1 \mathrm{~Hz}$ QPO in EXO 0748-676 as a function of $S_{\mathrm{a}}$. From top to bottom we show frequency, $Q$-value, and fractional rms. The errors on the frequency are typically smaller than the symbol size. The 20 gray horizontal bars in the upper panel show the boundaries between intervals used for grouping power spectra based on QPO frequency (see main text).

the power spectra at low $S_{\mathrm{a}}$. In Figure 5 we show three representative power spectra, in which the $\sim 1 \mathrm{~Hz}$ QPO has frequencies of $\sim 0.5, \sim 1.4$, and $\sim 6.6 \mathrm{~Hz}$ (top, middle, and bottom panel, respectively). Fits to these power spectra are shown as well, including the individual components (a power law and up to five Lorentzians). As can be seen, in all cases the $\sim 1 \mathrm{~Hz}$ QPO is accompanied by several broad components and a power-law noise component. Based on a comparison with other NS-LMXBs (see Section 3.2) and following the nomenclature used in prior works, we identify the following components in the power spectrum in the top panel of Figure 5: a break $\left(L_{\mathrm{b}}\right)$, the $\sim 1 \mathrm{~Hz}$ QPO, which we identify as a LF-QPO $\left(L_{\mathrm{LF}}\right)$, a "hump" $\left(L_{\mathrm{h}}\right)$, and two broad features that are commonly associated with the lower and upper $\mathrm{kHz}$ QPOs $\left(L_{1}\right.$ and $\left.L_{\mathrm{u}}\right)$. Given the quality of the power spectra, the lower and upper $\mathrm{kHz}$ QPOs are typically not well constrained and they will not be discussed in detail. The fit parameters of the $\sim 1 \mathrm{~Hz}$ QPO $\left(L_{\mathrm{LF}}\right)$ and the two low-frequency noise components $\left(L_{\mathrm{b}}\right.$ and $\left.L_{\mathrm{h}}\right)$ are given in Table 1 . The power-law component gradually steepened from $\alpha \approx 1.5$ at low QPO frequencies to $\alpha \approx 2.5$ at high $\mathrm{QPO}$ frequencies, while it decreased in strength from $\sim 7 \%-8 \%$ to $\sim 2 \%-3 \%$.

In Figure 6 we once again show the evolution of the $\sim 1 \mathrm{~Hz}$ QPO properties as a function of $S_{\mathrm{a}}$, this time using the measurements from the frequency-selected groups. As can be seen from the top panel, combining groups based on frequency greatly reduces the scatter at low $S_{\text {a }}$ compared to Figure 4, although some scatter is still present. Also, for the same reason

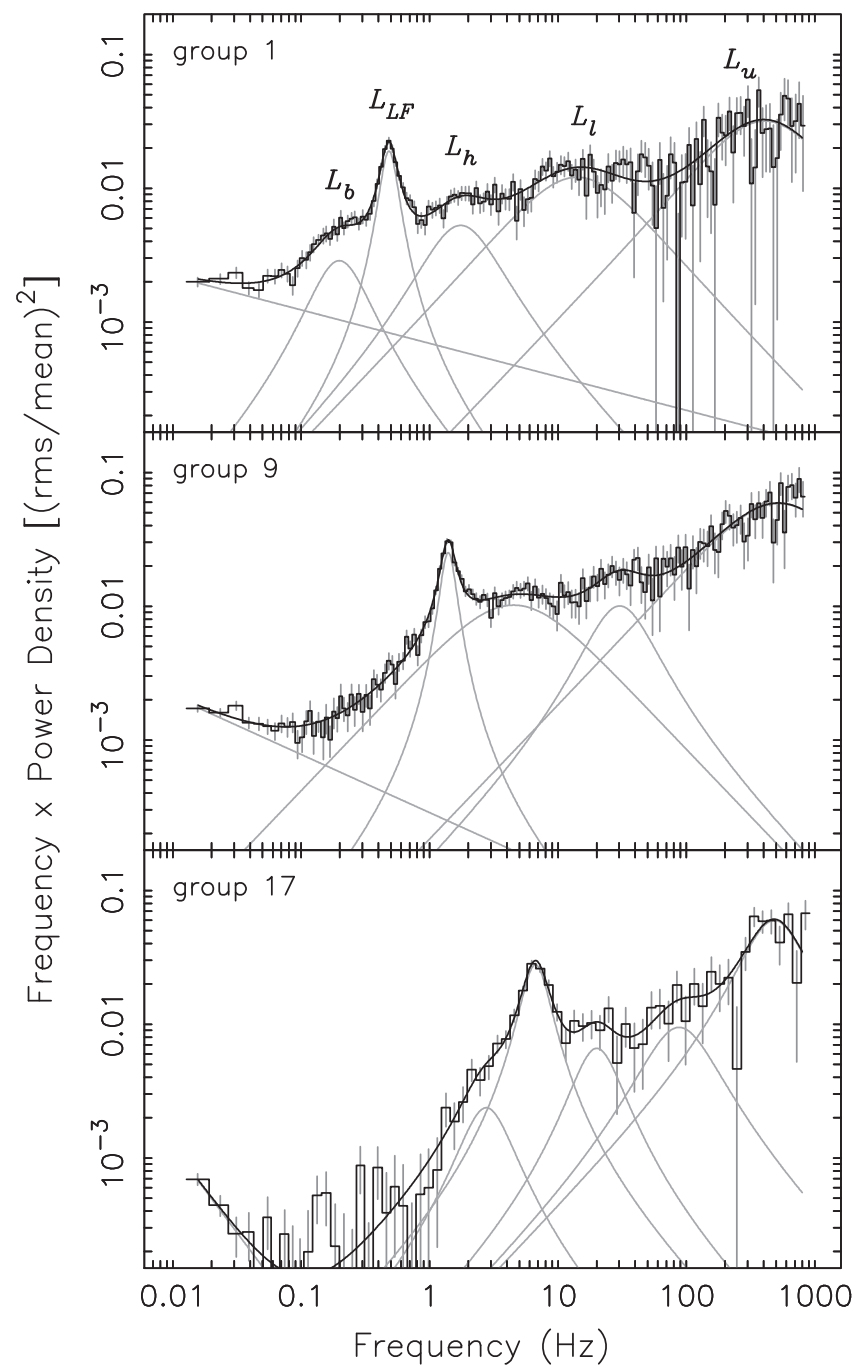

Figure 5. Three representative power spectra of EXO 0748-676 from frequency-selected groups. Fits to the power spectra are shown as solid black curves, while individual components are shown in gray. The various powerspectral components are identified in the top panel. The $\sim 1 \mathrm{~Hz}$ QPO is marked as $L_{\mathrm{LF}}$.

no data points are present any longer below $S_{\mathrm{a}}=1.09$. As before, the QPO frequency shows a strong increase with $S_{\mathrm{a}}$. The $Q$-value decreases slowly up to $S_{\mathrm{a}} \approx 1.4-1.6$ and appears to increase after that. Although the fractional rms amplitude of the QPO still shows considerable scatter at low $S_{\mathrm{a}}$ values, overall there is an increasing trend up to $S_{\mathrm{a}} \approx 1.4$. The rms appears to drop again toward higher $S_{\mathrm{a}}$ values.

The $L_{\mathrm{h}}$ noise component is detected in all frequency-selected power spectra, except for the last one. The $L_{\mathrm{b}}$ noise component is consistently detected up to QPO frequencies of $\sim 1.2 \mathrm{~Hz}$. At higher QPO frequencies the $L_{\mathrm{h}}$ component becomes broader and as a result the $L_{\mathrm{b}}$ component becomes more difficult to detect. It is only detected twice more, when the $L_{\mathrm{h}}$ component is narrower.

\subsection{Comparison with Other Atoll Sources}

Since EXO 0748-676 shows atoll-like patterns in its CD and HID, we investigated whether the $\sim 1 \mathrm{~Hz}$ QPO in EXO 0748-676 is possibly related to the LF-QPOs seen in the non-dipping/eclipsing atoll sources. Due to their relatively 
Table 1

Power Spectral Fit Parameters

\begin{tabular}{|c|c|c|c|c|c|c|c|c|c|c|}
\hline \multirow[b]{2}{*}{ No. ${ }^{a}$} & \multirow[b]{2}{*}{$S_{\mathrm{a}}$} & \multicolumn{3}{|c|}{$L_{\mathrm{LF}}$} & \multicolumn{3}{|c|}{$L_{\mathrm{b}}$} & \multicolumn{3}{|c|}{$L_{\mathrm{h}}$} \\
\hline & & $\begin{array}{l}\nu_{\max } \\
(\mathrm{Hz})\end{array}$ & Q & $\begin{array}{l}\mathrm{rms} \\
(\%)\end{array}$ & $\begin{array}{l}\nu_{\max } \\
(\mathrm{Hz})\end{array}$ & Q & $\begin{array}{l}\mathrm{rms} \\
(\%)\end{array}$ & $\begin{array}{l}\nu_{\max } \\
(\mathrm{Hz})\end{array}$ & $Q$ & $\begin{array}{l}\text { rms } \\
(\%)\end{array}$ \\
\hline 1 & $1.142 \pm 0.003$ & $0.483 \pm 0.003$ & $2.9 \pm 0.2$ & $9.8 \pm 0.3$ & $0.20 \pm 0.11$ & $0.83 \pm 0.19$ & $6.5 \pm 0.7$ & $1.75 \pm 0.13$ & $0.58 \pm 0.19$ & $9.8 \pm 1.3$ \\
\hline 2 & $1.116 \pm 0.003$ & $0.574 \pm 0.004$ & $3.1 \pm 0.3$ & $8.9 \pm 0.4$ & $0.19 \pm 0.16$ & $0.7 \pm 0.3$ & $5.9 \pm 0.9$ & $2.1 \pm 0.3$ & $0.2 \pm 0.2$ & $12.9_{-1.5}^{+2.4}$ \\
\hline 3 & $1.122 \pm 0.003$ & $0.665 \pm 0.003$ & $3.5 \pm 0.2$ & $11.1 \pm 0.3$ & $0.27 \pm 0.19$ & $0.8 \pm 0.2$ & $6.7 \pm 0.8$ & $2.11 \pm 0.16$ & $0.34 \pm 0.15$ & $14.8 \pm 1.3$ \\
\hline 4 & $1.158 \pm 0.001$ & $0.749 \pm 0.003$ & $3.14 \pm 0.17$ & $10.9 \pm 0.3$ & $0.27 \pm 0.16$ & $0.8 \pm 0.2$ & $5.1 \pm 0.6$ & $2.25 \pm 0.10$ & $0.42 \pm 0.09$ & $15.8 \pm 1.1$ \\
\hline 5 & $1.090 \pm 0.002$ & $0.857 \pm 0.003$ & $3.36 \pm 0.16$ & $10.2 \pm 0.2$ & $0.31 \pm 0.15$ & $1.7 \pm 0.4$ & $3.5 \pm 0.4$ & $2.66 \pm 0.18$ & $0.23 \pm 0.07$ & $16.8 \pm 1.0$ \\
\hline 6 & $1.143 \pm 0.001$ & $0.952 \pm 0.004$ & $3.4 \pm 0.2$ & $12.3 \pm 0.4$ & $0.3 \pm 0.2$ & $1.4_{-0.4}^{+0.7}$ & $4.0 \pm 0.7$ & $2.8 \pm 0.2$ & $0.30 \pm 0.08$ & $18.2 \pm 1.2$ \\
\hline 7 & $1.146 \pm 0.002$ & $1.040 \pm 0.004$ & $3.5 \pm 0.19$ & $10.2 \pm 0.3$ & $0.40 \pm 0.03$ & $1.4_{-0.4}^{+0.7}$ & $3.3 \pm 0.7$ & $3.2 \pm 0.3$ & $0.23 \pm 0.10$ & $15.7 \pm 1.7$ \\
\hline 8 & $1.200 \pm 0.002$ & $1.182 \pm 0.007$ & $3.1 \pm 0.2$ & $10.2 \pm 0.4$ & $0.50 \pm 0.05$ & $0.9_{-0.5}^{+0.9}$ & $4.3 \pm 1.3$ & $3.4 \pm 0.3$ & $0.38 \pm 0.19$ & $13.9_{-1.3}^{+2.0}$ \\
\hline 9 & $1.175 \pm 0.002$ & $1.397 \pm 0.007$ & $3.25 \pm 0.19$ & $10.7 \pm 0.3$ & $\cdots$ & $\cdots$ & $\cdots$ & $4.5 \pm 0.5$ & $0.04 \pm 0.08$ & $17.6 \pm 0.9$ \\
\hline 10 & $1.167 \pm 0.002$ & $1.598 \pm 0.009$ & $2.92 \pm 0.18$ & $11.6 \pm 0.3$ & $\cdots$ & $\ldots$ & $\cdots$ & $5.7 \pm 0.7$ & $0^{\mathrm{b}}$ & $18.3 \pm 0.8$ \\
\hline 11 & $1.209 \pm 0.002$ & $1.901 \pm 0.013$ & $3.1 \pm 0.3$ & $10.3 \pm 0.4$ & $\cdots$ & $\cdots$ & $\cdots$ & $4.5 \pm 1.2$ & $0.09 \pm 0.12$ & $15.3_{-0.5}^{+1.6}$ \\
\hline 12 & $1.261 \pm 0.002$ & $2.430 \pm 0.014$ & $2.76 \pm 0.19$ & $13.2 \pm 0.5$ & $1.09_{-0.18}^{+0.47}$ & $0.6 \pm 0.3$ & $5.7 \pm 1.9$ & $7.4 \pm 0.7$ & $0.3 \pm 0.2$ & $15.4 \pm 1.4$ \\
\hline 13 & $1.298 \pm 0.005$ & $2.84 \pm 0.05$ & $2.1 \pm 0.3$ & $11.0 \pm 0.7$ & $\ldots$ & $\ldots$ & $\ldots$ & $7.1_{-1.2}^{+1.8}$ & $0^{\mathrm{b}}$ & $13.3 \pm 1.0$ \\
\hline 14 & $1.303 \pm 0.003$ & $3.75 \pm 0.03$ & $2.9 \pm 0.3$ & $11.6 \pm 0.5$ & $\cdots$ & $\cdots$ & $\cdots$ & $8.0 \pm 1.0$ & $0^{\mathrm{b}}$ & $14.9 \pm 0.9$ \\
\hline 15 & $1.322 \pm 0.005$ & $4.61 \pm 0.05$ & $2.9 \pm 0.3$ & $12.5 \pm 0.6$ & $\cdots$ & $\cdots$ & $\cdots$ & $7.6 \pm 1.2$ & $0^{\mathrm{b}}$ & $15.3 \pm 1.0$ \\
\hline 16 & $1.361 \pm 0.009$ & $5.43 \pm 0.11$ & $2.9 \pm 0.6$ & $12.7 \pm 1.1$ & $\cdots$ & $\cdots$ & $\cdots$ & $15_{-5}^{+8}$ & $0^{\mathrm{b}}$ & $13.1_{-1.0}^{+2.5}$ \\
\hline 17 & $1.370 \pm 0.003$ & $6.68 \pm 0.10$ & $1.8 \pm 0.3$ & $14.7 \pm 0.9$ & $2.8 \pm 0.9$ & $1.0_{-0.4}^{+0.8}$ & $5.6_{-1.3}^{+2.5}$ & $20 \pm 3$ & $1.0_{-0.6}^{+0.9}$ & $9.1_{-1.3}^{+2.9}$ \\
\hline 18 & $1.407 \pm 0.004$ & $8.96 \pm 0.16$ & $2.3 \pm 0.4$ & $12.9 \pm 0.9$ & $\ldots$ & $\ldots$ & $\ldots$ & $16 \pm 3$ & $0^{\mathrm{b}}$ & $18.0 \pm 1.1$ \\
\hline 19 & $1.516 \pm 0.005$ & $12.8 \pm 0.2$ & $2.5 \pm 0.5$ & $12.0 \pm 1.0$ & $\cdots$ & $\cdots$ & $\cdots$ & $22 \pm 8$ & $0^{\mathrm{b}}$ & $13.1_{-0.9}^{+2.0}$ \\
\hline 20 & $1.631 \pm 0.009$ & $17.0 \pm 0.4$ & $4.6_{-1.5}^{+2.6}$ & $9.4 \pm 1.5$ & $\cdots$ & $\cdots$ & $\cdots$ & $28_{-12}^{+45}$ & $0^{\mathrm{b}}$ & $13.6_{-1.2}^{+4.8}$ \\
\hline 21 & $1.729 \pm 0.015$ & $25.3 \pm 1.2$ & $3.5 \pm 1.6$ & $11.3 \pm 1.7$ & $\cdots$ & $\cdots$ & $\cdots$ & $\cdots$ & $\cdots$ & $\cdots$ \\
\hline
\end{tabular}

Notes.

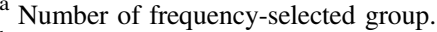

${ }^{\mathrm{b}}$ Value was fixed.

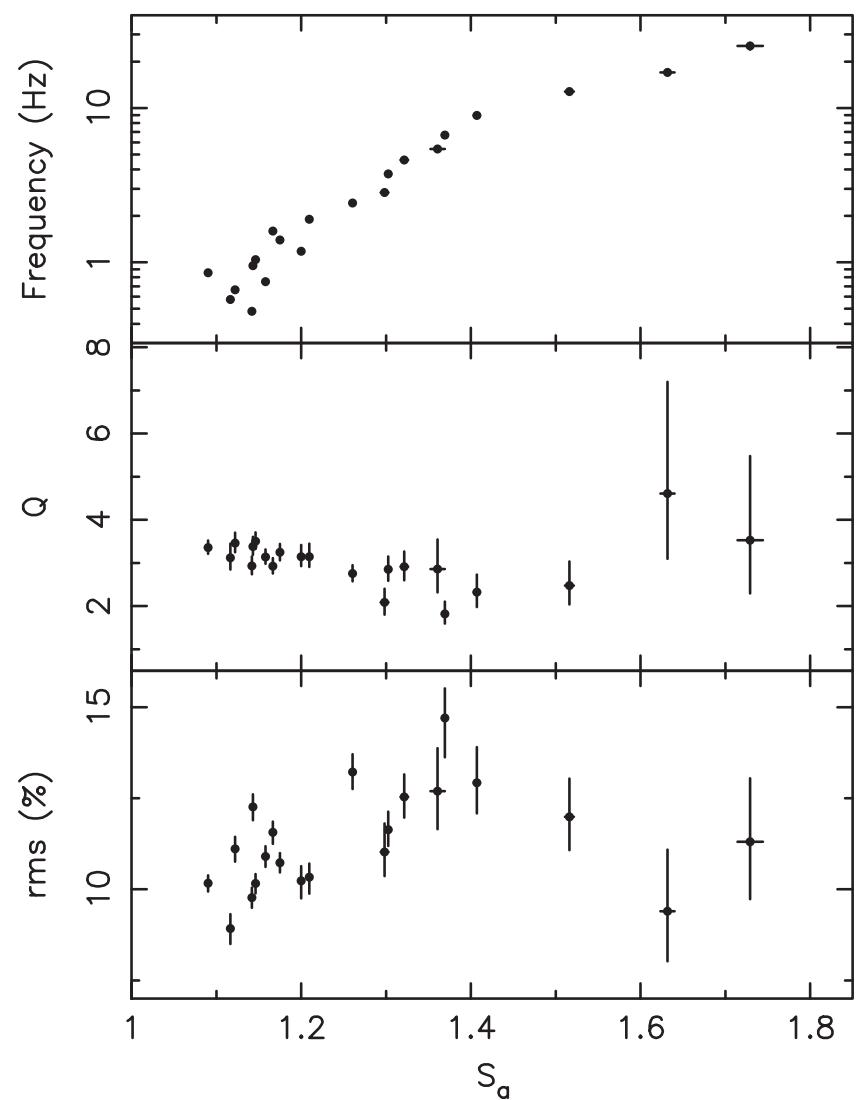

Figure 6. Properties of the $\sim 1 \mathrm{~Hz}$ QPO in EXO 0748-676 as a function of $S_{\mathrm{a}}$. From top to bottom we show frequency, $Q$-value, and fractional rms. The errors on the frequency are typically smaller than the symbol size. low rms amplitudes ( few percent) detections of LF-QPOs in atoll sources are not very common (especially compared to the LF-QPOs in the more luminous $\mathrm{Z}$ sources). We first searched the literature for $R X T E$ observations of non-dipping/eclipsing atoll sources with LF-QPOs that have frequency ranges (partially) overlapping that of the $\sim 1 \mathrm{~Hz}$ QPO in EXO 0748-676. Several examples were found: 4U 1636-53 (Altamirano et al. 2008a), 4U 1608-52 (van Straaten et al. 2003), 1E 1724-3045 (Altamirano et al. 2008b), and 4U 1820-30 (Altamirano et al. 2005). In addition we also analyzed observations of 4U 1812-12 and found LF-QPOs around $0.3 \mathrm{~Hz}$. In Figure 7 we compare the power spectra of the first three of these atoll sources with three power spectra of EXO 0748-676.

For similar QPO frequencies we find that the power spectra of EXO 0748-676 and the three atoll sources are to a large extent similarly shaped, with the accompanying noise and (broad) QPO components having similar frequencies as well. Most importantly, like the $\sim 1 \mathrm{~Hz}$ QPO in EXO 0748-676, the LF-QPO component in the three atoll sources has a frequency between that of the $L_{\mathrm{b}}$ and $L_{\mathrm{h}}$ noise components (although in 1E 1724-3045 it is closer to the $L_{\mathrm{h}}$ component than in the other sources). However, there are several differences as well. The $\sim 1 \mathrm{~Hz}$ QPO in EXO 0748-676 is considerably stronger than the LF-QPOs detected in the atoll sources (in the same frequency range). As can be seen in Table 1 , the $\sim 1 \mathrm{~Hz}$ QPO in EXO 0748-676 has a fractional rms amplitude in the range $\sim 9 \%-15 \%$. The atoll source LF-QPOs, on the other hand, have fractional rms amplitudes of $\sim 2 \%-5 \%$. On average the $Q$ values of the atoll sources LF-QPOs tend to be higher than those of the $\sim 1 \mathrm{~Hz}$ QPO in EXO 0748-676; this is especially 

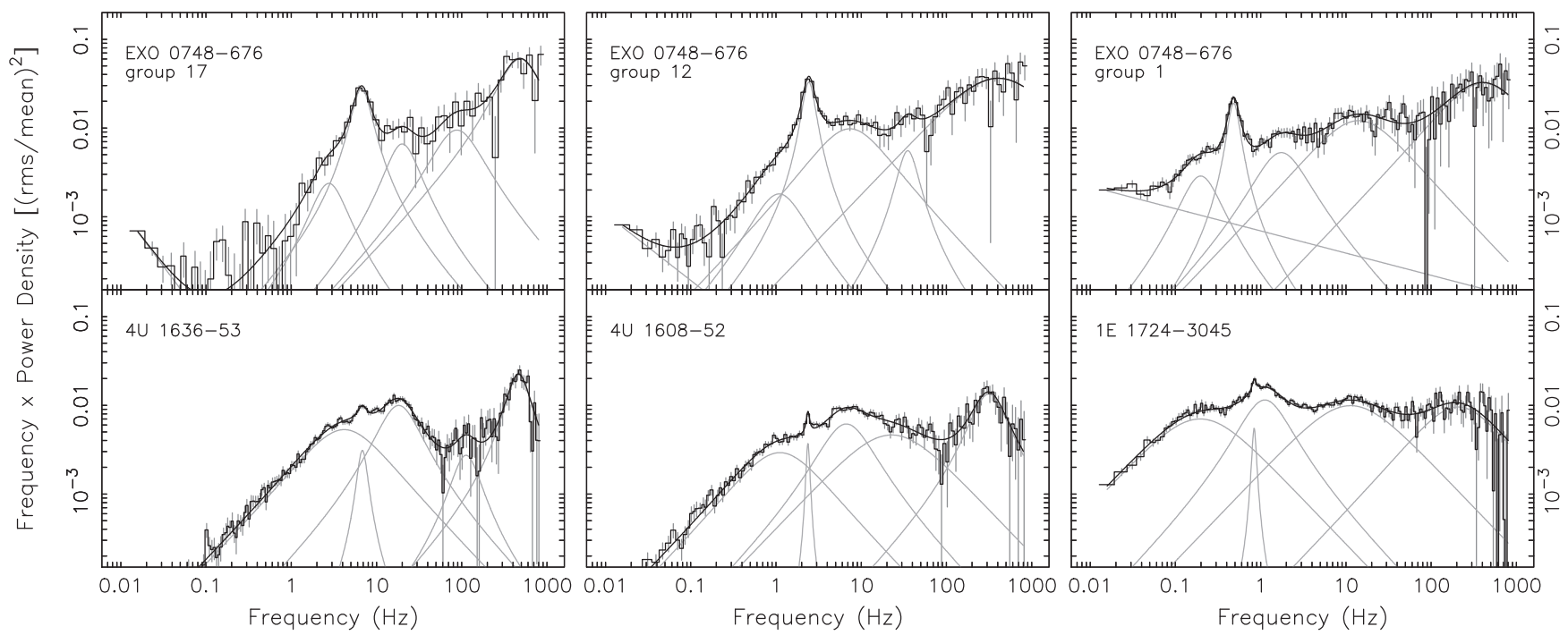

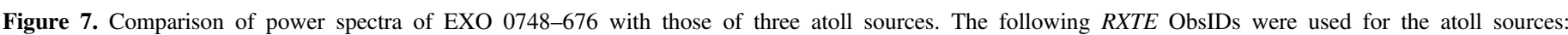
60032-05-21-00(0) for 4U 1636-53, 30062-02-02-00(0) for 4U 1608-52, and 10090-01-01-000 for 1E 1724-3045.

clear for the examples shown in Figure 7. In EXO 0748-676 the $Q$-values ranged from $\sim 1.8$ to $\sim 4.6$, while in the atoll source values of $\sim 1.6-8.8$ are found (see above references). While grouping of observations may have led to some broadening of the QPO in EXO 0748-676, similar $Q$-values were already reported for QPO detections in individual observations by Homan et al. (1999).

There are some differences in the behavior of the other power spectral components as well. The power spectra of EXO 0748-676 contain a clear power-law noise component, which is absent in the atoll source power spectra. Although some atoll sources do show such a power-law component in their power spectra, it typically does not appear until the source is closer to the soft state (see, e.g., van Straaten et al. 2003). We note that strong power-law components are present in some of the other dipping and/or eclipsing sources as well (Jonker et al. 1999, 2000), suggesting its presence is related to viewing angle. Another difference pertains to the two broad high-frequency components (which we tentatively identified as the lower and upper $\mathrm{kHz}$ QPOs); these tend to be stronger in EXO 0748-676 than in the other atoll sources, as can be seen from Figure 7.

As the $\sim 1 \mathrm{~Hz}$ QPO in EXO 0748-676 and LF-QPOs in the atoll sources appear to have similar frequencies relative to those of the two main $\left(L_{\mathrm{b}}\right.$ and $\left.L_{\mathrm{h}}\right)$ noise components, it is interesting to explore whether the QPOs also follow similar relations in QPO frequency versus noise frequency diagrams. Such studies have been performed for various power-spectral components in NS-LMXBs and BH-LMXBs (see, e.g., Psaltis et al. 1999; Wijnands \& van der Klis 1999; Belloni et al. 2002). In Figure 8 we plot the frequency of the QPOs in EXO 0748-676 and several atoll sources as a function of the frequency of the break component $L_{\mathrm{b}}$ (left panel) and as a function of the frequency of the hump component $L_{\mathrm{h}}$ (right panel). In addition to the atoll sources mentioned earlier, we also include data from the dipping NS-LMXB EXO 1745-248 (stars), which also shows a $\sim 1 \mathrm{~Hz}$ QPO (Homan 2012). For this source the break frequency was poorly constrained. Finally, for reference, we show in the left panel of Figure 8 the original data of Wijnands \& van der Klis (1999) for the Z and atoll sources. We note, however, that for the power spectra of the atoll sources
Wijnands \& van der Klis (1999) mostly used the frequency of $L_{\mathrm{h}}$ for that of the QPO. As a result the QPO values that we plot for EXO 0748-676 and the atoll sources fall well below the relation found by Wijnands \& van der Klis (1999).

As can be seen from both panels in Figure 8, EXO 0748-676 traces out relations that are largely consistent with those of the other atoll sources. We note that 1E 1724-3045 (gray squares) presents somewhat of an outlier in both panels. The QPO frequency for this source lies a factor of $\sim 2$ above that of the relation traced out by the other sources, suggesting that the QPO in 1E 1724-3045 might be the second harmonic, with the fundamental not being present. In $4 \mathrm{U}$ 1812-12 (triangles) and EXO 1745-248 (stars) we detect the fundamental as well as the second harmonic. Both are shown in Figure 8, with the fundamentals falling on the main relation and the second harmonics coinciding with the cluster of 1E 1724-3045 data points. This appears to confirm the harmonic nature of the $1 \mathrm{E}$ 1724-3045 QPO. For this reason we also show the QPO frequencies of 1E 1724-3045 divided by a factor of two in Figure 8 (red squares); as can be seen, in this way the 1E 1724-3045 data line up well with the main relation.

\section{DISCUSSION}

We have presented a study of the $\sim 1 \mathrm{~Hz}$ QPO in the dipping and eclipsing NS-LMXB EXO 0748-676. The main goal of this work was to understand how this QPO evolves between the various spectral states and how its properties compare to those of the LF-QPOs in other NS-LMXBs.

In its HID and CD EXO 0748-676 traces out tracks that strongly resemble those of the atoll source NS-LMXBs, with hard (extreme island), transitional (island), and soft (banana) states. We found that the $\sim 1 \mathrm{~Hz}$ QPO in EXO 0748-676 is only observed in the hard and intermediate states and that its frequency strongly increases as the spectrum softens. After combining power spectra based on QPO frequency, a relatively tight relation with the track parameter $S_{\mathrm{a}}$ is observed (see Figure 6), with the frequency increasing from $\sim 0.5 \mathrm{~Hz}$ at $S_{\mathrm{a}} \approx$ 1.2 to $\sim 25 \mathrm{~Hz}$ at $S_{\mathrm{a}} \approx 1.8$. This increase in frequency from the hard state into the intermediate state, as well as the frequency range covered, is similar to what is observed for the LF-QPOs 

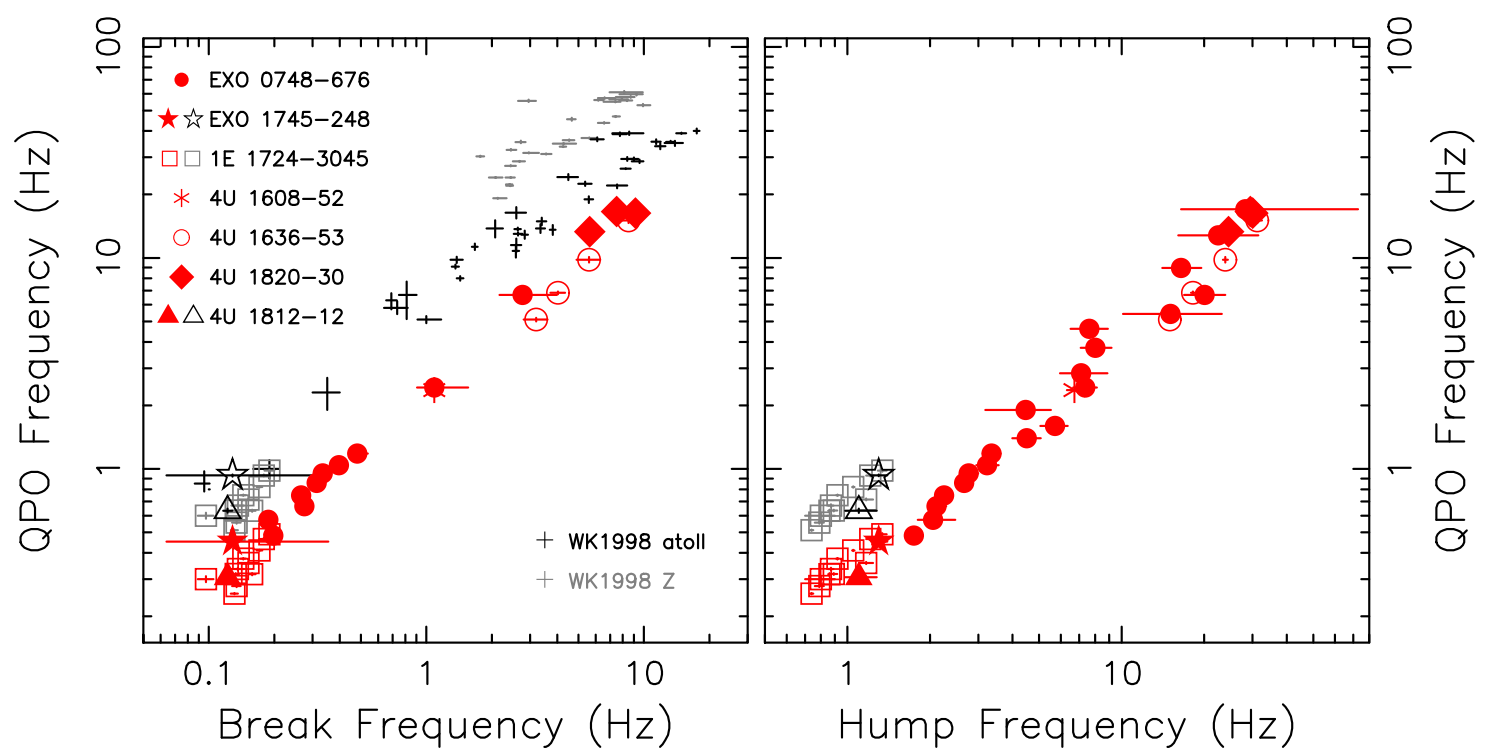

Figure 8. QPO frequency vs. break frequency (left panel) and vs. hump frequency (right panel). See the left panel for the meaning of the various symbols. For reference we also show the original data from Wijnands \& van der Klis (1999) for atoll and Z sources. For 1E 1724-3045 (gray open squares) we also show the QPO frequencies divided by a factor of two (red open squares). Data were taken from Altamirano et al. (2008a) for 4U 1636-53, Altamirano et al. (2005) for 4U 1820-30, and Altamirano et al. (2008b) for 1E 1724-3045. For 4U 1608-52 we analyzed RXTE observations 30062-02-02-00(0), for 4U 1812-12 observations 30701-13-01-[00:02], and for EXO 1745-248 observation 50054-06-04-03.

in other atoll sources in these states (van Straaten et al. 2003; Altamirano et al. 2005, 2008a, 2008b).

The grouping of power spectra from individual ObsIDs (first based on time and later also based on QPO frequency) resulted in high-quality power spectra that revealed several peaked noise components in addition to the QPO and power-law noise component already reported in the $\sim 1 \mathrm{~Hz}$ QPO discovery papers. A comparison of the QPO and noise components in the power spectra of EXO 0748-676 with those in atoll source power spectra revealed strong overall similarities. Most importantly, like the LF-QPO in atoll sources, the $\sim 1 \mathrm{~Hz}$ QPO in EXO 0748-676 $\left(L_{\mathrm{LF}}\right)$ is located between the break $\left(L_{\mathrm{b}}\right)$ and hump $\left(L_{\mathrm{h}}\right)$ noise components (Figure 7$)$ and its frequency evolves in a similar way with respect to that of the two noise components (Figure 8).

These results strongly suggest that the frequencies of the $\sim 1 \mathrm{~Hz}$ QPO in EXO 0748-676 and the LF-QPOs in (other) atoll sources are set by the same mechanism. From past work (Homan et al. 1999; Jonker et al. 1999, 2000) it is clear that $\sim 1 \mathrm{~Hz}$ QPOs in EXO 0748-676 and other dipping/eclipsing NS-LMXBs have a geometric nature, in the sense that parts of the accretion flow continuously move (rotate) in and out of our line of sight to the neutron star, thus modulating the emission we see from the neutron star surface and/or boundary layer. Homan (2012) suggested that a misaligned precessing inner flow could provide such a continuously changing geometry.

Since the non-dipping/eclipsing atoll sources have lower inclination angles, and there is no evidence for line of sight modulations, such a precessing inner disk would have to modulate the X-ray emission in a different manner in those sources. Modulation of the X-rays through relativistic beaming and light-bending effects, as suggested for LF-QPOs in BHLMXBs (Schnittman et al. 2006), could offer an explanation for the non-dipping/eclipsing sources. We note that these effects are likely also at work in dipping/eclipsing sources. However, Figure 3 in Schnittman et al. (2006) suggests that the resulting modulations are out of phase with the modulations caused by the line of sight interceptions. This may in fact reduce the overall strength of the $\sim 1 \mathrm{~Hz}$ QPO, although it is still observed to be significantly stronger than the LF-QPOs in non-dipping/eclipsing atoll sources. The differences in modulation mechanism between the two types of QPOs may also explain the (on average) higher rms amplitudes and lower quality factors of the $\sim 1 \mathrm{~Hz}$ QPOs in comparison to the LFQPOs in the non-dipping/eclipsing atoll sources, although detailed simulations are necessary to verify this. In addition to resulting in different modulation mechanisms for the QPOs, we speculate that the higher inclination of EXO 0748-676 may also be the cause of the more prominent power-law noise component as well as differences in the relative strengths of the broad peaked noise components, although the mechanisms involved are unclear.

As mentioned in Section 1, there is increasing evidence for a geometric origin of the (type-C) LF-QPOs in BH-LMXBs (Heil et al. 2015; Ingram \& van der Klis 2015; Motta et al. 2015), with Lense-Thirring precession (Stella \& Vietri 1998) currently being the most promising mechanism (Ingram et al. 2009). While we argued above that the $\sim 1 \mathrm{~Hz}$ QPO in EXO 0748-676 and the LF-QPOs in other atoll sources have a geometric nature as well, it is not clear to what extent their frequencies are set, or dominated, by Lense-Thirring precession. Classical precession (due to neutron-star oblateness) and magnetic precession (due to magnetic torques) may significantly lower the precession frequency (Lai 1999; Shirakawa \& Lai 2002), while radiation feedback from the neutron star could significantly increase the precession frequency (Miller 1999). The latter mechanism may explain why, for the same break $\left(L_{\mathrm{b}}\right)$ frequency, the LF-QPOs in the more luminous $\mathrm{Z}$ sources (the horizontal-branch oscillations) have higher frequencies (by a factor of $\sim 5$, see Figure 8 ) than the $\sim 1 \mathrm{~Hz}$ QPO and LFQPOs in EXO 0748-676 and other atoll sources. In fact, Altamirano et al. (2012) already pointed out that the frequencies of the horizontal-branch oscillations in the $\mathrm{Z}$ source and $11 \mathrm{~Hz}$ pulsar IGR J17480-2446 were too high to be 
explained by pure Lense-Thirring precession, suggesting that radiation feedback can indeed significantly alter (or even dominate) the precession frequency in neutron star systems. However, we stress that the conclusions we have drawn about the geometric nature of the $\sim 1 \mathrm{~Hz}$ QPO and LF-QPOs in atoll sources do not necessarily apply to the horizontal-branch oscillations in the $\mathrm{Z}$ sources since these conclusions were based on the similarities between the power spectra of EXO 0748-676 and the non-dipping/eclipsing atoll sources. The horizontal-branch oscillations follow a very different relation in the left panel of Figure 8 (although with a similar slope) and it is therefore not clear whether they are the result of a similar mechanism as the LF-QPOs in atoll sources.

In none of the seven NS-LMXBs that have shown $\sim 1 \mathrm{~Hz}$ QPOs are the QPOs detected in the soft state (Homan 2012). Combined with the fact that LF-QPOs in non-dipping/ eclipsing atoll sources are also rare (or absent) in the soft state, this suggests that the precessing part of the accretion flow in atoll NS-LMXBs becomes significantly smaller toward the soft state and/or that the misalignment angle between the inner flow and neutron-star spin axis decreases. Both changes would reduce the effects of line of sight interceptions in the dipping/ eclipsing systems and of the relativistic beaming and lightbending effects in non-dipping/eclipsing sources. In the case of the $\sim 1 \mathrm{~Hz}$ QPO a reduction in the geometrical thickness and/or the optical thickness of the inner flow may also affect the efficiency of removing photons from the neutron star out of our line of sight and therefore reduce the strength of the QPO. Finally, the frequency increase of the $\sim 1 \mathrm{~Hz}$ QPOs in EXO 0748-676 and the LF-QPOs in the atoll sources indicates that the transition radius between the (standard) accretion disk and the precessing inner accretion flow decreases as the spectrum softens, similar to what has been concluded from the type-C QPOs in BH-LMXBs (Ingram \& Done 2011).

\section{CONCLUSIONS}

We have studied the $\sim 1 \mathrm{~Hz}$ QPO in the dipping and eclipsing NS-LMXB EXO 0748-676 and compared it to the LF-QPOs in non-dipping/eclipsing atoll NS-LMXBs. We find strong similarities between the two types of QPOs and conclude that their frequencies are likely set by the same mechanism. A misaligned precessing inner accretion flow is suggested as the common frequency mechanism; the resulting modulations are geometric in nature, but the way in which the modulations occur depends on viewing angle. In the (lowinclination) non-dipping/eclipsing sources the emission from the precessing flow could be modulated through relativistic beaming and light-bending effects, while in the (higherinclination) dipping/eclipsing systems we get an additional (and dominating) modulation of the X-rays from the neutron star through line of sight interceptions by the precessing inner flow.

J.H. acknowledges financial support from NASA grant NNX12AE14G, provided through the Astrophysics Data Analysis Program. This research has made use of data obtained from the High Energy Astrophysics Science Archive Research Center (HEASARC), provided by NASA's Goddard Space Flight Center.

\section{REFERENCES}

Altamirano, D., Ingram, A., van der Klis, M., et al. 2012, ApJL, 759, L20 Altamirano, D., van der Klis, M., Méndez, M., et al. 2005, ApJ, 633, 358 Altamirano, D., van der Klis, M., Méndez, M., et al. 2008a, ApJ, 685, 436 Altamirano, D., van der Klis, M., Méndez, M., et al. 2008b, ApJ, 687, 488 Belloni, T., \& Hasinger, G. 1990, A\&A, 227, L33

Belloni, T., Psaltis, D., \& van der Klis, M. 2002, ApJ, 572, 392

Bradt, H. V., Rothschild, R. E., \& Swank, J. H. 1993, A\&AS, 97, 355

Casella, P., Belloni, T., \& Stella, L. 2005, ApJ, 629, 403

Dieters, S. W., \& van der Klis, M. 2000, MNRAS, 311, 201

Fridriksson, J. K. 2011, PhD thesis, Massachusetts Institute of Technology

Fridriksson, J. K., Homan, J., \& Remillard, R. A. 2015, ApJ, 809, 52

Gladstone, J., Done, C., \& Gierliński, M. 2007, MNRAS, 378, 13

Heil, L. M., Uttley, P., \& Klein-Wolt, M. 2015, MNRAS, 448, 3348

Homan, J. 2012, ApJL, 760, L30

Homan, J., Jonker, P. G., Wijnands, R., van der Klis, M., \& van Paradijs, J. 1999, ApJL, 516, L91

Homan, J., \& van der Klis, M. 2000, ApJ, 539, 847

Ingram, A., \& Done, C. 2011, MNRAS, 415, 2323

Ingram, A., Done, C., \& Fragile, P. C. 2009, MNRAS, 397, L101

Ingram, A., \& van der Klis, M. 2015, MNRAS, 446, 3516

Jahoda, K., Markwardt, C. B., Radeva, Y., et al. 2006, ApJS, 163, 401

Jonker, P. G., van der Klis, M., Homan, J., et al. 2000, ApJ, 531, 453

Jonker, P. G., van der Klis, M., \& Wijnands, R. 1999, ApJL, 511, L41

Lai, D. 1999, ApJ, 524, 1030

Levine, A. M., Bradt, H., Cui, W., et al. 1996, ApJL, 469, L33

Miller, J. M., \& Homan, J. 2005, ApJL, 618, L107

Miller, M. C. 1999, ApJ, 520, 256

Miyamoto, S., Kimura, K., Kitamoto, S., Dotani, T., \& Ebisawa, K. 1991, ApJ, 383,784

Motta, S. E., Casella, P., Henze, M., et al. 2015, MNRAS, 447, 2059

Muñoz-Darias, T., Fender, R. P., Motta, S. E., \& Belloni, T. M. 2014, MNRAS, 443, 3270

Ponti, G., Muñoz-Darias, T., \& Fender, R. P. 2014, MNRAS, 444, 1829

Psaltis, D., Belloni, T., \& van der Klis, M. 1999, ApJ, 520, 262

Remillard, R. A., Lin, D., Cooper, R. L., \& Narayan, R. 2006, ApJ, 646, 407

Schnittman, J. D., Homan, J., \& Miller, J. M. 2006, ApJ, 642, 420

Shirakawa, A., \& Lai, D. 2002, ApJ, 564, 361

Stella, L., \& Vietri, M. 1998, ApJL, 492, L59

van der Klis, M. 1995, in Proc. NATO Advanced Study Institute on the Lives of the Neutron Stars, ed. M. A. Alpar, U. Kiziloglu, \& J. van Paradijs (Dordrecht: Kluwer Academic), 301

van Straaten, S., van der Klis, M., \& Méndez, M. 2003, ApJ, 596, 1155

Wijnands, R., \& van der Klis, M. 1999, ApJ, 514, 939

Wolff, M., Ray, P., Wood, K., \& Wijnands, R. 2008a, ATel, 1812

Wolff, M. T., Ray, P. S., \& Wood, K. S. 2008b, ATel, 1736

Wolff, M. T., Ray, P. S., Wood, K. S., \& Hertz, P. L. 2009, ApJS, 183, 156 